\title{
Uso de três princípios de intervenção aumenta a efetividade da terapia por contensão induzida: estudo de caso
}

\section{Use of three principles of intervention increases the effectiveness of constraint induced therapy: case report}

\author{
Natalia Duarte Pereira' ${ }^{1,}$ Isabella de Souza Menezes ${ }^{2}$, \\ Sarah Monteiro dos Anjos ${ }^{3}$
}

\begin{abstract}
PEREIRA, N. D.; MENEZES, I. S.; ANJOS, S. M. Uso de três princípios de intervenção aumenta a efetividade da Terapia por Contensão Induzida: estudo de caso. Rev. Ter. Ocup. Univ. São Paulo, v. 21, n. 1 , p. 33-40, jan./abr. 2010.

RESUMO: Terapia por Contensão Induzida (TCI) é formada por três componentes: treino repetitivo de tarefa orientada, restrição da extremidade do membro superior menos acometido e da aplicação de um conjunto de métodos comportamentais de reforço de adesão para transferir os ganhos obtidos na clinica para o ambiente real do paciente. Porém apenas dois desses princípios são aplicados com freqüência. O objetivo deste estudo foi de caracterizar o protocolo de intervenção através de um relato longitudinal de caso de um paciente com seqüela de hemiparesia crônica após AVE. O paciente foi avaliado pela Motor Activity Log (MAL) e pelo Wolf Motor Function Test (WMFT) e os resultados sugerem que os aspectos comportamentais pertencentes a técnica têm grande influência nos bons resultados e a TCI pode ter maior ação nas atividades de vida diária de pacientes com hemiplegia quando aplicados todos os três tipos de intervenção em que consiste.
\end{abstract}

DESCRITORES: Acidente cerebral vascular. Extremidade superior. Hemiplegia. Terapia por exercício. Estudos de caso.

1 Fisioterapeuta especialista em Neurologia, mestre em Ciências do Movimento Humano pela Universidade Estadual de Santa Catarina com treinamento em Constraint Induced Movement Therapy na Universidade do Alabama em Birmingham - EUA.

2 Fisioterapeuta da Associação de Assistência à Criança Deficiente especialista em Neurologia com treinamento Constraint Induced Movement Therapy na Universidade do Alabama em Birmingham - EUA.

3 Terapeuta Ocupacional da Associação de Assistência à Criança Deficiente especialista em Tecnologia Assistiva com treinamento Constraint Induced Movement Therapy na Universidade do Alabama em Birmingham - EUA.

Endereço para correspondência: Natalia Duarte Pereira. Rua Madrigal, n 86. Condomínio Monte Belo. Salto-SP. CEP:13320-410. natalia@contensaoinduzida.com.br 
PEREIRA, N. D. et al. Uso de três princípios de intervenção. Rev. Ter. Ocup. Univ. São Paulo, v. 21, n. 1, p. 33-40, jan./abr. 2010.

\section{INTRODUÇÃO}

$\mathrm{H}$

emiparesia é o déficit mais comum após um acidente vascular encefálico (AVE), afetando

mais de $80 \%$ das pessoas, agudamente e mais de $40 \%$, cronicamente. As técnicas de reabilitação têm tido mais sucesso em restaurar a função do membro inferior do que o membro superior, mas infelizmente, a função do membro superior é que dá uma maior independência nas atividades da vida diária e maior auto-estima ao indivíduo (LEVY et al., 2001, p. 4).

Uma estratégia de tratamento para promover melhora do uso do membro superior acometido é a Terapia por Contensão Induzida (TCI) ou Constraint-Induced Movement Therapy.

Esta técnica de tratamento é definida pelos criadores como um potente método de recuperação sensório-motora pós-AVE que desencoraja o uso do membro não afetado e encoraja o uso ativo do membro superior parético, tendo como objetivo maximizar ou restaurar a função motora (TAUB et al., 1993, p. 347).

Essa restauração é atribuída a uma reorganização cortical uso-dependente que interrompe um processo cíclico chamado não uso aprendido (MILTNER et al., 1999, p. 586).

A reorganização cortical uso dependente é um fenômeno de plasticidade cerebral onde o tamanho da área de representação cortical do membro superior por exemplo, é diretamente proporcional ao uso do mesmo. Esse fenômeno foi evidenciado em estudos (LIEPERT et al., 2000; LEVY et al., 2001; SCHAECHTER et al., 2002; PARK et al., 2004) que relatam alterações no padrão de atividade cerebral e aumento da área de representação cortical do membro superior acometido pelo AVE após a TCI (PARK et al., 2004, p. 95).

Schaechter et al. (2002, p. 326) em seu estudo de neuroimagem mostrou que a resposta de uma região cortical de um músculo da mão eliciada por ressonância magnética é quase o dobro depois da TCI em pacientes hemiplégicos crônicos comparado ao período pré tratamento.

Liepert et al. (2000, p. 1210) sugerem a possibilidade que o aumento do uso do membro mais afetado produzido pela TCI resulta em um aumento da representação cortical desse membro, que proporciona uma base neural para um aumento do uso do membro.

O projeto EXCITE (Extremity Constraint-Induced Therapy Evaluation) é um estudo clínico randomizado e multicêntrico que visa a melhora funcional dos membros superiores de pacientes com seqüela de hemiparesia após
AVE subagudos e crônicos. Este estudo teve como objetivo avaliar a capacidade da TCI em ampliar a capacidade funcional desses indivíduos, quando comparados àqueles que receberam tratamento convencional (WOLF et al., 2006, p. 296).

Uma revisão sistemática sobre o assunto concluiu que na maioria dos protocolos existentes observa-se ganho quando da aplicação da TCI comparando-se a outras intervenções ou ausência de intervenção (HAKKENNES; KEATING, 2005, p. 221).

São três os princípios ou intervenções que aplicados em conjunto, e só assim, formam a TCI: 1) Treino de tarefa orientada intensivo com repetição do membro superior mais acometido 3 horas por dia, por 2 semanas consecutivas (shaping e task pratice); 2) Restrição do membro superior menos afetado durante $90 \%$ das horas acordado no período do tratamento; 3) Aplicação de um conjunto de métodos comportamentais para reforço de adesão destinado a transferir os ganhos feitos no ambiente clínico para o mundo real do paciente (MORRIS et al., 2006, p. 257).

O treino de tarefa orientada, onde o movimento é organizado em torno de um objetivo motor e comportamental, é realizado de maneira repetitiva e intensiva. As tarefas propostas são ajustadas segundo as necessidades e objetivos de cada paciente proporcionando a solução ativa de problemas e adaptação às alterações ambientais que juntamente com o conhecimento do resultado somam alguns dos principais conceitos da aprendizagem motora e tornam o protocolo individualizado (SHUMWAY-COOK; WOOLLACOTT, 2003).

De acordo com Morris et al. (2006, p. 257), o shaping é um método de treinamento baseado nos princípios do treino comportamental, onde o objetivo motor é alcançado em pequenos passos. Diversos estudos apontam que as tarefas são flexíveis em relação à ampliação do nível de dificuldade e complexidade, de forma que os seus componentes podem ser modificados ao longo do protocolo de acordo com os parâmetros de progressão potenciais estabelecidos (TAUB et al., 1993, p. 347; MORRIS et al., 1997, p. 29; TAUB et al., 1999, p. 237; MORRIS et al., 2001, p.750). Segundo Bonifer e Anderson (2003, p. 384), a mudança na dificuldade de cada tarefa varia de acordo com a melhora do paciente. Para tanto, é importante definir como a tarefa será analisada estabelecendo qual o seu parâmetro de conhecimento dos resultados e quais movimentos devem ser enfatizados.

Já o task pratice é uma técnica menos estruturada, ou seja, envolve atividades funcionais que são realizadas 
PEREIRA, N. D. et al. Uso de três princípios de intervenção. Rev. Ter. Ocup. Univ. São Paulo, v. 21, n. 1, p. 33-40, jan./abr. 2010.

integralmente, em um período de 15 a 30 minutos (MORRIS et al., 2006, p. 257).

A aplicação dos métodos para reforço de adesão, também chamado de métodos para transferência, é realizada durante o protocolo da TCI e inclui procedimentos que são usados freqüentemente em intervenções comportamentais para modificação de hábitos. No protocolo original criado por Taub, os métodos propostos são um contrato formal de comprometimento para o uso da luva, uma lista de tarefas para casa e um diário de casa.

Nos primeiros estudos sobre TCI foi utilizado uma tipóia para restrição do membro superior não afetado, porém esse tipo de aparato limitava as reações de equilíbrio além de não restringir o uso da mão. Em 2002, em um estudo com 15 pacientes Sterr e colaboradores propuseram o uso de uma luva para pacientes com problemas de equilíbrio e da tipóia para os demais. Não foi observada diferença nos resultados entre os grupos. Desde então uma luva que limita a preensão mas permite o apoio tem sido utilizada para as pesquisas.

Como é possível observar, a restrição do membro superior não é o principal componente da técnica, e sim apenas um lembrete para que o paciente não use o membro sadio ou menos afetado em casa. $\mathrm{O}$ aparato não apresenta outra função, já que é apenas uma luva removível que garante a segurança do paciente (WOLF, 2007, p. 1212).

E é por isso que optamos pelo termo "Contensão" (grande aplicação ou esforço para resolver uma dificuldade segundo o Dicionário Básico da Língua Portuguesa) para o nome da técnica, pois essa conotação está de acordo com seus princípios básicos da que vão muito além da contenção (restrição) do membro superior mais acometido.

No Brasil já existem alguns estudos publicados com a aplicação da técnica e embora praticamente todos os trabalhos de TCI apresentarem resultados positivos, a variabilidade desses resultados é ampla (RIBERTO et al., 2005; SOUSA, 2008; VAZ et al., 2008).

Uma possível razão para essa variação é que alguns pesquisadores utilizaram apenas um ou dois em vez de todos três componentes da TCI.

Por exemplo, Vaz et al. (2008, p. 298) realizou TCI em um indivíduo com hemiparesia utilizando apenas a restrição e o treino repetitivo de tarefa, não incluindo os métodos comportamentais de transferência de uso do membro superior mais acometido para o ambiente real.

Outra possível explicação é que alguns pesquisadores não aplicam a intensidade do tratamento como proposto pelos criadores da técnica. Como descreveu van der
Lee et al. (1999, p. 2369) sobre o treinamento de uso hemiparéticos em um formato de grupo, utilizando "Atividades de limpeza, artesanato, música e jogos," que é provável que resultaram em uso muito menos intenso do que é desejável para a TCI (TAUB et al., 2006, p. 1045). No Brasil também temos um exemplo no estudo de Sousa em 2008 onde além do uso do aparato de restrição, foram realizadas apenas duas sessões de fisioterapia convencional por semana.

Este estudo de um único caso tem como objetivo comparar os resultados da intervenção original proposta por Taub do protocolo de TCI com os resultados obtidos no país com protocolos modificados.

\section{Procedimentos Metodológicos}

O estudo foi realizado na Associação de Assistência a Criança Deficiente no período de Outubro de 2009 a Fevereiro de 2010 com aprovação do comitê de ética da instituição com protocolo número: 039/2010.

Um sujeito do sexo masculino, com 62 anos de idade com hemiparesia à direita decorrente de um AVE hemorrágico participou do estudo após 15 meses de lesão e foi selecionado pelo método não probabilístico e intencional.

Foram suspensas as sessões de fisioterapia e terapia ocupacional no período do estudo. Como critério de inclusão era necessário possuir escore maior ao ponto de corte de acordo com a escolaridade no Mini Exame do Estado Mental (BERTOLUCCI et al., 1994, p. 1). Além disso o participante deveria apresentar movimentação ativa de no mínimo 45 graus de flexão e abdução de ombro, 20 graus de extensão de cotovelo, 10 graus de extensão de punho, 10 graus de abdução/extensão do polegar e ter pelo menos 10 graus de extensão em mais dois dedos além do polegar (WINSTEIN et al., 2003, p. 137).

Para avaliação da movimentação ativa, foram solicitadas três repetições de cada movimento no intervalo de tempo de um minuto com o antebraço apoiado e o punho posicionado na beirada de uma mesa (WINSTEIN et al., 2003, p. 137).

Em seguida foi submetido à avaliação pela Motor Activity Log (MAL), uma entrevista estruturada que avalia em escalas de 5 pontos a quantidade e a qualidade com que o paciente usa o seu membro superior mais afetado fora do ambiente terapêutico em 30 atividades do seu dia a dia. E pelo Wolf Motor Function Teste (WMFT) que tem 
PEREIRA, N. D. et al. Uso de três princípios de intervenção. Rev. Ter. Ocup. Univ. São Paulo, v. 21, n. 1, p. 33-40, jan./abr. 2010.

como objetivo avaliar a habilidade motora dos membros superiores de pacientes com hemiparesia. A avaliação com o WMFT contêm 17 tarefas sendo duas delas avaliação de força. Neste estudo foram realizadas as 15 tarefas de acordo com o manual disponibilizado pela Universidade do Alabama que são cronometradas.

Após a avaliação foi iniciado o protocolo de intervenção com os três fundamentos básicos da técnica por duas semanas consecutivas. No final da segunda semana o paciente foi reavaliado assim como após um e três meses do término do protocolo para acompanhamento dos resultados.

As técnicas dos métodos comportamentais usadas para reforço de adesão destinado a transferir os ganhos feitos no ambiente clínico para o mundo real do paciente foram: contrato de comprometimento de uso da luva, diário de casa e lista de tarefas.

O contrato de comprometimento é um acordo formal que foi realizado no primeiro dia do tratamento onde terapeuta, paciente e cuidador estabeleceram após um levantamento das tarefas diárias do paciente, em quais atividades do dia seria permitido a retirada da restrição e o uso das duas mãos ou auxílio do cuidador. Todos assinaram o contrato e o paciente levou uma cópia para casa para consulta caso fosse necessário.

A lista de 10 tarefas foi formada pelas atividades que o paciente teve menor pontuação na escala da qualidade da MAL. Todos os dias a lista foi conferida, pois o paciente anotou quais das atividades propostas ele tentou usar o membro superior mais afetado e por quanto tempo.

O diário de casa é um relatório que o paciente fez sobre suas atividades assim que deixava o ambiente terapêutico até retornar ao mesmo no dia seguinte. Nesse diário constava o uso ou não da luva para cada atividade realizada. O objetivo dessa medida foi além de checar o tempo de uso da luva, proporcionar a solução ativa de problemas ou seja, o paciente trouxe para a sessão as dificuldades encontradas e junto com a terapeuta foram propostas soluções potenciais (TAUB et al., 2006, p. 1045).

Foi orientado também para o paciente e sua família que o lado afetado seja usado o maior número de vezes possível em suas atividades de vida diária e que o uso da luva fosse feito por $90 \%$ das horas que o paciente permanecesse acordado. Porém em atividades de higiene básica, como o banho, ou que ofereçam risco para o paciente, foi aconselhada a retirada da restrição. Também por motivo de segurança a restrição indicada foi uma luva, pois esta impede de maneira mais efetiva o uso da mão não acometida e permite o uso dos membros para reações de equilíbrio e proteção.

A intervenção se caracteriza pelo treino intensivo, portanto foram realizadas três horas diárias de aplicação das técnicas de shaping e task pratice. Em cada sessão eram aplicados 4 shapings e um task pratice selecionados entre um banco de tarefas propostas pela Universidade do Alabama e de acordo com os componentes de movimento mais comprometidos do membro superior mais afetado do paciente (MORRIS et al., 2006, p. 258).

Após a escolha da tarefa do shaping era selecionado o parâmetro de progressão para que com pequenos ajustes no ambiente ou no objeto da tarefa, a mesma fosse se tornando mais desafiadora, porém sempre possível para que a frustração do paciente não ocorresse. Dessa maneira eram realizadas atividades funcionais segmentadas, com enfoque em um componente de movimento que foi realizado em 10 repetições de 30 a 45 segundos cada. Foi cronometrado o tempo de cada uma das 10 repetições, além disso o terapeuta dava uma nota de qualidade de execução dada pelo terapeuta em uma escala de 0 a 5 . Os resultados eram apresentados ao paciente para que a diminuição no tempo e/ou aumento na qualidade de realização da tarefa servisse como motivador para continuação do treino. Ao final da sessão uma tarefa funcional do task pratice era escolhida para que o componente de movimento treinado fosse inserido no contexto da atividade.

\section{RESULTADOS}

A Tabela 1 apresenta os resultados da MAL pré, pós tratamento e no acompanhamento de 1 e 3 meses.

Das 30 atividades questionadas através da MAL no pré tratamento, apenas seis eram realizadas segundo o paciente. No pós tratamento eram realizadas 28 atividades entre as 30 questionadas. Mesmo após 3 meses do término da TCI as atividades continuavam sendo realizadas em casa e com grande aumento na escala da qualidade de 2,42 no pré para 3,48 após 3 meses.

A Tabela 2 apresenta a pontuação obtida no WMFT antes e após o tratamento com TCI.

Houve uma melhora em relação a média de tempo que o paciente levou para completar as tarefas do WMFT de 7,06 para 6,6 segundos. 
PEREIRA, N. D. et al. Uso de três princípios de intervenção. Rev. Ter. Ocup. Univ. São Paulo, v. 21, n. 1, p. 33-40, jan./abr. 2010.

Tabela 1 - Resultados obtidos de acordo com a Motor Activity Log (MAL) no pré, pós tratamento e reavaliações de 1 e 3 meses

\begin{tabular}{|c|c|c|c|c|c|c|c|c|}
\hline & \multicolumn{4}{|c|}{ Quantidade de movimento } & \multicolumn{4}{|c|}{ Qualidade de movimento } \\
\hline & pré & pós & 1 mês & 3 meses & pré & pós & 1 mês & 3 meses \\
\hline ligar a luz no interruptor & 2 & 3 & 3 & 3 & 3 & 3 & 4 & 4 \\
\hline abrir uma gaveta & 0 & 3 & 3 & 2,5 & - & 4 & 3 & 3 \\
\hline tirar item de roupa da gaveta & 0 & 3 & 2 & 3 & - & 4 & 4 & 4 \\
\hline pegar um telefone & 0 & 0 & 3 & 1,5 & - & & 3 & 3 \\
\hline limpar um balcão de cozinha & 0 & 4 & 2 & 3,5 & - & 3 & 3 & 4 \\
\hline sair do carro & 3 & 2 & 3 & 3 & 3 & 4 & 3 & 4 \\
\hline abrir a geladeira & 0 & 3 & 3 & 4 & - & 4 & 4 & 4 \\
\hline abrir a porta com a maçaneta & 0 & 3 & 2 & 3 & - & 3 & 3 & 3 \\
\hline usar um controle remoto & 1,5 & 4 & 4 & 2 & 2 & 4 & 4 & 4 \\
\hline lavar as mãos & 3,5 & 4 & 4 & 4 & 3,5 & 5 & 5 & 5 \\
\hline abrir e fechar torneiras & 0 & 2 & 2 & 3 & - & 3 & 2 & 3,5 \\
\hline secar as mãos & 0 & 4 & 4 & 4 & - & 5 & 5 & 4 \\
\hline colocar meia & 0 & 2 & 2 & 3 & - & 2 & 2 & 3 \\
\hline tirar as meias & 0 & 2 & 2 & 3 & - & 2 & 2 & 3,5 \\
\hline colocar sapatos & 0 & 2 & 2 & 3,5 & - & 2 & 2 & 3 \\
\hline tirar sapatos & 0 & 3 & 2 & 3 & - & 3 & 2 & 3 \\
\hline levantar de uma cadeira de braços & 0 & 4 & 3 & 3,5 & - & 4 & 3 & 4 \\
\hline puxar a cadeira para se sentar & 0 & 4 & 2 & 4 & - & 4 & 4 & 4 \\
\hline puxar a cadeira para perto da mesa & 0 & 4 & 4 & 2,5 & - & 4 & 3,5 & 4 \\
\hline pegar um copo, garrafa... & 0 & 3 & 3 & 3 & - & 4 & 4 & 3 \\
\hline escovar os dentes & 0 & 0 & 0 & 2 & - & & & 2 \\
\hline passar maquiagem, loção ou creme & 0 & 4 & 2 & 2 & - & 3 & 3 & 3 \\
\hline chave para trancar a porta & 0 & 2 & 2 & 2,5 & - & 2 & 2 & 3 \\
\hline escrever no papel & 0 & 2 & 0 & 5 & - & 2 & & 2,5 \\
\hline carregar um objeto & 2 & 4 & 3 & 4 & 2 & 4 & 4 & 4 \\
\hline usar um garfo ou colher & 0 & 2 & 3 & 2 & - & 3 & 3 & 3 \\
\hline pentear os cabelos & 0 & 2 & 4 & 2 & - & 2 & 3 & 3 \\
\hline pegar uma xícara pela alça & 0 & 3 & 3 & 3 & - & 3 & 4 & 4 \\
\hline abotoar a camisa & 2 & 2 & 3 & 3,5 & 1 & 2 & 4 & 3 \\
\hline $\begin{array}{l}\text { comer com as mãos (bolachas, } \\
\text { lanche) }\end{array}$ & 0 & 4 & 3 & 4 & - & 4 & 4 & 4 \\
\hline Média & 0,65 & 2,8 & 2,6 & $\mathbf{3 , 0 7}$ & 2,42 & 3,29 & 3,3 & 3,48 \\
\hline
\end{tabular}

Escala de quantidade de movimento (QT), Escala de qualidade de movimento (QL). Escores variando de 0 (não usa o membro) a 5 (usa tanto quanto e com a mesma qualidade de antes do AVE). 
PEREIRA, N. D. et al. Uso de três princípios de intervenção. Rev. Ter. Ocup. Univ. São Paulo, v. 21, n. 1, p. 33-40, jan./abr. 2010.

Tabela 2 - Resultado da avaliação pelo Wolf Motor Function Test (WMFT) em cada tarefa realizada com o membro superior mais afetado pré e pós tratamento

\begin{tabular}{lcc}
\hline & Tempo Pré TCI (seg.) & Tempo Pós TCI (seg.) \\
\hline Antebraço na mesa & 3,35 & 1,94 \\
Antebraço na caixa & 2,10 & 3,38 \\
Extensão do cotovelo & 1,68 & 1,40 \\
Extensão do cotovelo c/ peso & 1,31 & 1,28 \\
Mão na mesa & 3,00 & 2,31 \\
Mão na caixa & 1,50 & 2,44 \\
Alcançar e retroceder & 1,00 & 1,90 \\
Levantar lata & 5,69 & 5,72 \\
Levantar lápis & 20,34 & 25,00 \\
Levantar clipe & 13,78 & 7,62 \\
Empilhar peças & 11,31 & 8,09 \\
Virar cartas & 11,00 & 13,00 \\
Virar chave & 15,22 & 11,00 \\
Dobrar toalha & 8,28 & 8,97 \\
Levantar cesta & 6,40 & 4,94 \\
Média & $\mathbf{7 , 0 6}$ & $\mathbf{6 , 6}$ \\
\hline tempo máximo de realização de cada tarefa era de 120 segundos. & \\
& & \\
\hline & & \\
& &
\end{tabular}

\section{DISCUSSÃO}

$\mathrm{O}$ aumento de mais de dois pontos na escala da quantidade da MAL sugerem um maior uso do membro superior mais afetado durante as atividades de vida diária. Essas alterações na MAL, particularmente da escala de quantidade, são comparáveis aos resultados publicados do projeto multicêntrico EXCITE (PAGE et al., 2008, p. 551).

Uma relação entre esse aumento do uso mantido após a TCI e uma reorganização cortical uso dependente tem sido relatada em artigos que associaram a aplicação da MAL com técnicas de representação cortical.

Como exemplo disso, Johansen-Berg et al. (2002, p. 2731), afirmaram que uma mudança comportamental no uso do membro superior acometido de pacientes adultos com hemiparesia está associada ao aumento de atividade do córtex pré motor e somatossensorial contralateral. Esse aumento do uso do membro superior acometido chamado de mudança comportamental também estaria associado a diminuição da ativação do córtex pré motor do membro superior não acometido pela diminuição do uso do mesmo.

Já a inserção desses movimentos e inclusive do membro superior nas atividades de vida diária vista através dos bons resultados da MAL pode ser atribuída aos componentes do método de transferência, porque devido a resolução de problemas e tarefas para casa o paciente pode ultrapassar barreiras no uso do membro superior em seu ambiente.

A transferência dos ganhos obtidos em tratamento, segundo Winstein (1999, p. 181) são maiores quando as demandas da prática clínica se assemelham ao ambiente real do paciente. Com os métodos comportamentais de reforço de adesão ao tratamento grande parte da prática é realizada pelo paciente no ambiente real e isso se reflete nos resultados da TCI.

Miltner et al. (1999, p. 586) encontrou um comportamento semelhante aos resultados apresentados da MAL que mesmo após seis meses do término da terapia, 
continuaram aumentando.

A estrutura sistemática de repetição nas tarefas do shaping foi útil na capacitação do participante para a prática das tarefas completas do task pratice, pois ajudou em movimentos específicos e necessários para melhorar a função do membro superior. Bowman et al. (2006, p. 167) também tiveram esse resultado de transferência do movimento repetido no shaping para o task pratice no estudo de caso realizado com um paciente com hemiparesia após AVE submetido ao protocolo completo de intervenção, ou seja, com os três princípios da técnica.

O WMFT tem apresentado diminuição discreta no seu tempo médio de execução em estudos do Excite e em uma discussão sobre as possíveis razões para isso foram levantadas algumas hipóteses. Uma delas seria que alguns pacientes já realizam a tarefa em um tempo razoável e a melhora após a TCI seria somente na qualidade de execução da tarefa. Outra hipótese é que o aumento do uso do membro superior mais acometido em alguns pacientes não se dá pelo aumento de velocidade de execução de alguns movimentos. A inclusão desse membro se daria então pela participação intensa nas atividades de vida diária durante a TCI atingida principalmente através do conjunto de métodos para transferência (BLANTON et al., 2006, p. 1520).

Outros fatores importantes que colaboraram para os resultados atingidos foram a assiduidade do paciente na terapia, a colaboração da família facilitando o uso do membro superior mais acometido nas tarefas de casa. Apesar da grande demanda do protocolo de intervenção que além das três horas diárias exige tarefas a serem realizadas em casa, não houve desistência nem falta de comprometimento do paciente. Acredita-se que essa motivação se deu pelo tempo determinado em duas semanas de protocolo e pelos resultados serem apresentados a cada dia para o paciente.

Os resultados apresentados nesse estudo são de apenas um paciente e portanto faz-se necessário que um maior número de pacientes sejam submetidos a técnica nos mesmos moldes para uma melhor avaliação dessa efetividade.

\section{CONCLUSÕES}

Assim podemos concluir que os aspectos comportamentais pertencentes a técnica parecem ter grande influência nos bons resultados e a Terapia de Contensão Induzida tem um impacto maior nas atividades de vida diária de pacientes com hemiplegia quando aplicados todos os três tipos de intervenção em que consiste.

Através das informações metodológicas deste estudo espera-se que a técnica seja divulgada na íntegra em nosso país, para que os pesquisadores e clínicos interessados possam dar continuidade a seu trabalho de modo que os resultados obtidos no Brasil sejam comparáveis aos da literatura mundial pela equivalência da intervenção proposta.

PEREIRA, N. D.; MENEZES, I. S.; ANJOS, S. M. Use of three principles of intervention increases the effectiveness of Constraint Induced Therapy: case report. Rev. Ter. Ocup. Univ. São Paulo, v. 21, n. 1, p. 33-40, jan./abr. 2010 .

\begin{abstract}
CIT has three components: repetitive task oriented training, restraining the less impaired extremity and applying a package of adherence enhancing behavioral methods designed to transfer gains made in the clinical setting to the patient's real world environment. But only two of these components are used with frequency. The aim of the present study was characterizing the intervention protocol through a longitudinal case report of a patient after stroke. The patient was evaluated by Motor Activity Log (MAL) and Wolf Motor Function Test (WMFT) and results suggest that behavioral aspects pertaining to technique have great influence on the success and TCI may have more action in the daily activities of patients with hemiplegia when applied to all three types of intervention that is.
\end{abstract}

KEY WORDS: Stroke. Hemiplegia. Upper extremity. Exercise therapy. Case studies.

\title{
REFERÊNCIAS
}

BERTOLUCCI, P., et al. O mini-exame do estado mental em uma população geral: impacto da escolaridade. Arq Neuropsiquiatr., v. 52, n. 1, p. 1-7, 1994.
BLANTON, S., et al.Lessons Learned in participant recruitment and retention: the EXCITE trial. Phys Ther., v. 86, n.11, p.15201533,2006 
PEREIRA, N. D. et al. Uso de três princípios de intervenção. Rev. Ter. Ocup. Univ. São Paulo, v. 21, n. 1, p. 33-40, jan./abr. 2010.

BONIFER, N.; ANDERSON, K. M. Application of constraintinduced movement therapy for an individual with severe chronic upper-extremity hemiplegia. Phys Ther., v. 83, p.384-398, 2003.

BOWMAN, M. H. et al. A treatment for a chronic stroke patient with a plegic hand combining CI therapy with conventional rehabilitation procedures: case report. Neurorehabilitation, v. 21, p.167-176, 2006.

DROMERICK, A.; EDWARDS, D.; HAHN, M. Does the application of constraint-induced movement therapy during acute rehabilitation reduce arm impairment after ischemic stroke? Stroke, v. 31, p. 2984-2988, 2000.

HAKKENNES, S.; KEATING, J. L. Constraint-induced movement therapy following stroke: a systematic review of randomised controled trials. Aust. J. Physiother., v. 51, p. 221-231, 2005.

JOHANSEN-BERG, H., et al. Correlation between motor improvements and altered fMRI activity after rehabilitative therapy. Brain, v. 125, p. 2731-2742, 2002.

LEVY, C. E.; et al. Functional MRI evidence of cortical reorganization in upper-limb stroke hemiplegia treated with constraint-induced movement therapy. Am. J. Phys. Med. Rehabil., v. 80 , p. $4-12,2001$.

LIEPERT, J.; et al. Treatment-induced cortical reorganization after stroke in humans. Stroke, v. 31, p. 1210-1216, 2000.

MILTNER, W. H. R.; et al. Effects of constraint-induced movement therapy on patients with chronic motor deficits after stroke: a replication. Stroke, v. 30, n. 11, p. 586-592, 1999.

MORRIS, D. M.; et al. Constraint-induced (CI) movement therapy for motor recovery after stoke. Neurorehabilitation, v. 9, p. 2943, 1997.

MORRIS, D. M.; TAUB, E.; MARK, V. W. Constraint-induced movement therapy: characterizing the intervention protocol, Eura Medicophys, v. 42, p. 257-268, 2006.

MORRIS, D. M.; et al. The reliability of the wolf motor function test for assessing upper extremity function after stroke. Arch. Phys. Med. Rehabil., v. 82, p. 750-755, 2001.

PAGE, S.; et al. Modified constraint-induced therapy in chronic stroke: results of a single-blinded randomized controlled trial. Phys. Ther., v. 88, n. 3, p. 551-554, 2008.

PARK, S.; HORAK, F. B.; KUO, A. D. Postural feedback responses scale with biomechanical constraints in human standing. Exp. Brain Res., v. 154, p. 417-427, 2004.

RIBERTO, M.; et al. A terapia de restrição como forma de aprimoramento da função do membro superior em pacientes com hemiplegia. Acta Fisiatr., v. 12, n. 1, p.15-19, 2005.

SCHAECHTER, J. D.; et al. Motor recovery and cortical reorganization after constraint-induced movement therapy in stroke patients: a preliminary study. Neurorehabil. Neural. Rep., v. 16, p. 326-338, 2002.

SHUMWAY-COOK, A.; WOOLLACOTT, M. H.. Controle motor: teoria e aplicações práticas. 2a. ed. Barueri: Manole, 2003.

SOUSA, R. D.Terapia de restrição de membro superior não paretico e indução de movimento em pacientes hemiparéticos. 2008. 84f. Dissertação. Campinas: Universidade Estadual de Campinas, Faculdade de Ciências Médicas, 2008.

TAUB, E.; et al. NEPOMUCENO, C. S.; CONNELL, J. S.; CRAGO, J. E. Technique to improve chronic motor deficit after stroke. Arch. Phys. Med., v. 74, p. 347-354, 1993.

TAUB, E.; USWATTE, G.; PIDIKITI, R. Constraint-induced movement therapy: a new family of techniques with broad application to physical rehabilitation - a clinical review. J. Rehabil. Res. Develop., v. 36, p. 237-251, 1999.

TAUB, E.; et al. CHATTERJEE, A. Placebo-controlled trial of constraint-induced movement therapy for upper extremity after stroke. Stroke, v. 37, p. 1045-1049, 2006.

VAN DER LEE, J. H.; et al. Forced use of the upper extremity in chronic stroke patients: results from a single-blinded randomized clinical trial. Stroke, v. 30, p. 2369-2375, 1999.

VAZ, D. V., et al. Terapia de movimento induzido pela restrição na hemiplegia: um estudo de caso único. Fisioter. Pesq., v. 15, n. 3, p.298-303, 2008

WINSTEIN, C. J. Knowledge of results and motor learning: implications for physical therapy. In: Movement science. Alexandria: American Physical Therapy Association, 1999. v .1, p. 181-190.

WINSTEIN, C. J.; et al. Methods for a multisite randomized trial to investigate the effect of constraint-induced movement therapy in improving upper extremity function among adults recovering from a cerebrovascular stroke. Neurorehabil. Neural. Repair, v. 17, n. 3, p. 137-152, 2003.

WOLF, S. L.; WINSTEIN, C. J.; MILLER, J. P. Effect of constraint-induced movement therapy on upper extremity function 3 to 9 months after stroke: the EXCITE randomized clinical trial. JAMA, v. 296, p. 2095-2104, 2006.

WOLF, S. L. Revisiting constraintinduced movement therapy: are we too smitten with the mitten? Is all nonuse "learned"? and other quandaries. Phys. Ther., v. 7, p. 1212-1223, 2007.

WOLF, S. L.; et al. The Excite trial: relationship of intensity of constraint induced movement therapy to improvement in the wolf motor function test. Restorative Neurol. Neurosci., v. 25, p.549-562, 2007. 